Pacific

Journal of

Mathematics

\title{
A JANTZEN SUM FORMULA \\ FOR RESTRICTED VERMA MODULES OVER AFFINE KAC-MOODY ALGEBRAS AT THE CRITICAL LEVEL
}

JOHANNES KÜBEL 


\title{
A JANTZEN SUM FORMULA FOR RESTRICTED VERMA MODULES OVER AFFINE KAC-MOODY ALGEBRAS AT THE CRITICAL LEVEL
}

JOHANNES KÜBEL

\begin{abstract}
For a restricted Verma module of an affine Kac-Moody algebra at the critical level we describe the Jantzen filtration and calculate its character. This corresponds to the Jantzen sum formula of a baby Verma module over a modular Lie algebra. This also implies a new proof of the linkage principle which was already derived by Arakawa and Fiebig.
\end{abstract}

\section{Introduction}

To a simple complex Lie algebra $\mathfrak{g}$ with Cartan subalgebra $\mathfrak{h}$, one associates an affine Kac-Moody Lie algebra $\widehat{\mathfrak{g}}$ with Cartan subalgebra $\widehat{\mathfrak{h}}$. The root system $R$ of $\mathfrak{g}$ can be embedded into the root system $\widehat{R}$ of $\hat{\mathfrak{g}}$. Arakawa and Fiebig [2012a] introduced the category $\bar{\sigma}_{c}$ of restricted representations of $\hat{\mathfrak{g}}$ at the critical level. Denote by $O_{c}$ the direct summand of the usual highest weight category 0 over $\hat{\mathfrak{g}}$ which consists of modules with critical level. Then $\bar{\sigma}_{c}$ is the subcategory of $\mathrm{O}_{c}$ on which those elements of the Feigin-Frenkel center act by zero which are homogeneous of degree unequal to zero. We call $\overline{\mathrm{O}}_{c}$ the restricted category $\mathrm{O}$ and its objects restricted modules.

Conjecturally, the restricted category $\overline{\mathrm{O}}_{c}$ should have the same structure as the representation category over a small quantum group or a modular Lie algebra described in [Andersen et al. 1994]. The standard modules in $\bar{O}_{c}$, which should correspond to baby Verma modules in the representation categories of [Andersen et al. 1994], are the so called restricted Verma modules. They are the maximal restricted quotients of the ordinary Verma modules.

Towards the description of $\overline{\mathrm{O}}_{c}$, Arakawa and Fiebig [2012b] confirmed the above conjecture in the subgeneric case, and Frenkel [2005, Theorem 4.8] did so in the generic case. Andersen, Jantzen, and Soergel [Andersen et al. 1994, Chapter 6]

The author is supported by the DFG priority program 1388 .

MSC2010: 17B10, 17B67.

Keywords: restricted Verma modules at the critical level, Jantzen sum formula, affine Kac-Moody algebras, critical representations of affine Kac-Moody algebras, Jantzen filtration, category $\mathcal{O}$. 
and Kumar and Letzter [1997] computed a Jantzen sum formula for a baby Verma module $Z(\lambda)$ which describes the characters of the Jantzen filtration as an alternating sum formula of certain characters of baby Verma modules of weight "lower" than $\lambda$.

We deduce the analogous formula for restricted Verma modules at the critical level. To be more precise, let $\lambda \in \widehat{\mathfrak{h}}^{*}$ be a weight of critical level. We introduce the Jantzen filtration

$$
\bar{\Delta}(\lambda)=\bar{\Delta}(\lambda)^{0} \supset \bar{\Delta}(\lambda)^{1} \supset \bar{\Delta}(\lambda)^{2} \supset \cdots,
$$

and deduce the formula

$$
\sum_{i>0} \operatorname{ch} \bar{\Delta}(\lambda)^{i}=\sum_{\alpha \in R(\lambda)^{+}}\left(\sum_{i>0}\left(\operatorname{ch} \bar{\Delta}\left(\alpha \downarrow^{2 i-1} \lambda\right)-\operatorname{ch} \bar{\Delta}\left(\alpha \downarrow^{2 i} \lambda\right)\right)\right) .
$$

Here $R(\lambda)^{+} \subset R$ denotes the positive roots of the finite root system $R$ which are integral on $\lambda$. The notation $\alpha \downarrow^{i} \lambda$ for $i>0$ is defined inductively by $\alpha \downarrow\left(\alpha \downarrow^{i-1} \lambda\right)$, where $\alpha \downarrow \lambda=s_{\alpha} \cdot \lambda$ if $s_{\alpha} \cdot \lambda \leq \lambda$ and $\alpha \downarrow \lambda=s_{-\alpha+\delta} \cdot \lambda$ if $s_{\alpha} \cdot \lambda>\lambda$. Here $\delta \in \hat{R}$ denotes the smallest positive imaginary root and $s_{\alpha}, s_{-\alpha+\delta}$ are the reflections corresponding to the real roots $\alpha,-\alpha+\delta$ of the affine Weyl group $\widehat{\mathcal{W}}$ with its dot-action on $\widehat{\mathfrak{h}}^{*}$.

The strategy to prove the Jantzen sum formula is to deduce the subgeneric cases first and then put these together to get the general result in a very similar manner as in [Jantzen 1979, Chapters 5.6 and 5.7]. To deduce the subgeneric case we use a result of [Arakawa and Fiebig 2012b] which states that for $\lambda \in \widehat{\mathfrak{h}}^{*}$ critical and subgeneric the maximal submodule of $\bar{\Delta}(\lambda)$ is isomorphic to the simple module $L(\alpha \downarrow \lambda)$ with highest weight $\alpha \downarrow \lambda$.

Arakawa and Fiebig [2012a] introduced projective objects in the restricted category $\bar{\sigma}_{c}$ and a BGGH-reciprocity to deduce the linkage principle for restricted Verma modules conjectured by Feigin and Frenkel. It states that if the simple module with highest weight $\mu \in \widehat{\mathfrak{h}}^{*}$ appears as a subquotient in a Jordan-Hölder series of $\bar{\Delta}(\lambda)$, where $\lambda \in \widehat{\mathfrak{h}}^{*}$ is critical, then $\mu \in \widehat{\mathscr{W}}(\lambda) \cdot \lambda$. Here $\widehat{\mathscr{W}}(\lambda)$ denotes the integral affine Weyl group of the root system $\hat{R}(\lambda)$ corresponding to $\lambda$ with its dot-action on $\widehat{\mathfrak{h}}^{*}$.

The linkage principle immediately follows from the Jantzen sum formula and thus gives an independent proof.

\section{Preliminaries}

In this chapter we shortly introduce the construction of an (untwisted) affine Kac-Moody algebra starting with a simple Lie algebra. We collect some facts about the root data, the Weyl group and the invariant bilinear form. The results and definitions in this section can be found in [Kac 1990] and [Kac and Kazhdan 1979]. 
Affine Kac-Moody algebras. Let $\mathfrak{g}$ be a simple Lie algebra with a Borel subalgebra $\mathfrak{b}$ and a Cartan subalgebra $\mathfrak{h}$. We denote by $R$ the root system with positive roots $R^{+}$and by $\Pi$ the simple roots. Moreover, denote by ${ }^{\mathcal{W}}$ the finite Weyl group and by $\kappa: \mathfrak{g} \times \mathfrak{g} \rightarrow \mathbb{C}$ the Killing form.

We take a nonsplit central extension $\tilde{\mathfrak{g}}$ of the loop algebra $\mathfrak{g} \otimes \mathbb{C} \mathbb{C}\left[t, t^{-1}\right]$. As a vector space, $\tilde{\mathfrak{g}}$ is the direct sum $\left(\mathfrak{g} \otimes_{\mathbb{C}} \mathbb{C}\left[t, t^{-1}\right]\right) \oplus \mathbb{C} c$, where $c$ is a central element.

Adding a derivation operator $d$ with the property $[d, \cdot]=t(\partial / \partial t)$, we get the affine Kac-Moody algebra $\widehat{\mathfrak{g}}$ associated to $\mathfrak{g}$. As a vector space, we have $\widehat{\mathfrak{g}}=$ $\left(\mathfrak{g} \otimes_{\mathbb{C}} \mathbb{C}\left[t, t^{-1}\right]\right) \oplus \mathbb{C} c \oplus \mathbb{C} d$ and the Lie bracket is given by

$$
\begin{gathered}
{[c, \hat{\mathfrak{g}}]=\{0\},} \\
{\left[d, x \otimes t^{n}\right]=n x \otimes t^{n},} \\
{\left[x \otimes t^{n}, y \otimes t^{m}\right]=[x, y] \otimes t^{m+n}+n \delta_{m,-n} \kappa(x, y) c,}
\end{gathered}
$$

where $x, y \in \mathfrak{g}$ and $n \in \mathbb{Z}$. The Borel subalgebra of $\hat{\mathfrak{g}}$ corresponding to $\mathfrak{b} \subset \mathfrak{g}$ is given by

$$
\widehat{\mathfrak{b}}=\left(\mathfrak{g} \otimes_{\mathbb{C}} t \mathbb{C}[t]+\mathfrak{b} \otimes_{\mathbb{C}} \mathbb{C}[t]\right) \oplus \mathbb{C} c \oplus \mathbb{C} d,
$$

while the corresponding Cartan subalgebra of $\hat{\mathfrak{g}}$ is given by

$$
\widehat{\mathfrak{h}}=\mathfrak{h} \oplus \mathbb{C} c \oplus \mathbb{C} d .
$$

Affine roots, Weyl groups and bilinear forms. For a vector space $V$ we denote by $\langle\cdot, \cdot\rangle: V^{*} \times V \rightarrow \mathbb{C}$ the natural pairing with its dual space. Denote by $\leq$ the usual ordering on $\widehat{\mathfrak{h}}^{*}$; that is, $\lambda \leq \mu$ for $\lambda, \mu \in \widehat{\mathfrak{h}}^{*}$ if $\mu-\lambda$ can be expressed as a finite sum of positive roots. The projection $\widehat{\mathfrak{h}}=\mathfrak{h} \oplus \mathbb{C} c \oplus \mathbb{C} d \rightarrow \mathfrak{h}$ induces an embedding $\mathfrak{h}^{*} \subset \widehat{\mathfrak{h}}^{*}$. By this embedding we can consider all finite roots as elements of $\widehat{\mathfrak{h}}^{*}$. We define two weights $\Lambda_{\circ}, \delta \in \widehat{\mathfrak{h}}^{*}$ by the relations

$$
\begin{aligned}
\langle\delta, \mathfrak{h} \oplus \mathbb{C} c\rangle & =\{0\}, \\
\langle\delta, d\rangle & =1, \\
\left\langle\Lambda_{\circ}, \mathfrak{h} \oplus \mathbb{C} d\right\rangle & =\{0\}, \\
\left\langle\Lambda_{\circ}, c\right\rangle & =1 .
\end{aligned}
$$

Then the roots of $\widehat{\mathfrak{g}}$ with respect to $\widehat{\mathfrak{h}}$ are given by $\widehat{R}=\widehat{R}^{\mathrm{re}} \cup \widehat{R}^{\mathrm{im}}$, where

$$
\widehat{R}^{\text {re }}:=\{\alpha+n \delta \mid \alpha \in R \subset \widehat{R}, n \in \mathbb{Z}\}
$$

are the real roots, and

$$
\widehat{R}^{\mathrm{im}}:=\{n \delta \mid n \in \mathbb{Z}, n \neq 0\}
$$

are the imaginary roots. The positive roots $\widehat{R}^{+}$, that is, the roots of $\widehat{\mathfrak{b}}$ with respect 
to $\widehat{\mathfrak{h}}$, are then given

$$
\hat{R}^{+}=R^{+} \cup\{\alpha+n \delta \mid \alpha \in R, n>0\} \cup\{n \delta \mid n>0\} .
$$

Denote by $\theta$ the highest root of $R$. Then the set of simple affine roots is given by

$$
\widehat{\Pi}=\Pi \cup\{-\theta+\delta\} \subset \widehat{R}^{+} .
$$

For a real root $\alpha \in \widehat{R}^{\text {re }}$ we denote by $\alpha^{\vee} \in \widehat{\mathfrak{h}}$ its coroot which is uniquely defined by the properties $\alpha^{\vee} \in\left[\hat{\mathfrak{g}}_{\alpha}, \hat{\mathfrak{g}}_{-\alpha}\right]$ and $\left\langle\alpha, \alpha^{\vee}\right\rangle=2$.

We denote by $\widehat{\mathscr{W}} \subset \operatorname{Gl}\left(\widehat{\mathfrak{h}}^{*}\right)$ the affine Weyl group of the root system $\hat{R}$, which is the subgroup generated by the reflections $s_{\alpha}: \widehat{\mathfrak{h}}^{*} \rightarrow \widehat{\mathfrak{h}}^{*}, \lambda \mapsto \lambda-\left\langle\lambda, \alpha^{\vee}\right\rangle \alpha$, where $\alpha \in \widehat{R}^{\text {re }}$ is a real root. We can identify the finite Weyl group $\mathcal{W}$ with the subgroup of $\widehat{W}$ generated by the reflections $s_{\alpha}$ corresponding to finite roots $\alpha \in R$. Then $\mathscr{W}$ stabilizes the subspace $\mathfrak{h}^{*} \subset \widehat{\mathfrak{h}}^{*}$.

Let $\bar{\rho}:=\frac{1}{2} \sum_{\alpha \in R^{+}} \alpha$ be the half-sum of positive finite roots. We then set

$$
\rho:=\bar{\rho}+h^{\vee} \Lambda_{\circ},
$$

where $h^{\vee}$ is the dual Coxeter number of $\mathfrak{g}$. Then $\left\langle\rho, \alpha^{\vee}\right\rangle \neq 0$ for all $\alpha \in \widehat{R}^{\mathrm{re}}$ and $\langle\rho, c\rangle \neq 0$ as well. We can now define the $\rho$-shifted dot-action of $\widehat{\mathscr{W}}$ on $\widehat{\mathfrak{h}}^{*}$ by

$$
w \cdot \lambda:=w(\lambda+\rho)-\rho,
$$

where $w \in \widehat{\mathscr{W}}$ and $\lambda \in \widehat{\mathfrak{h}}^{*}$.

The Killing form $\kappa$ on the simple Lie algebra $\mathfrak{g}$ extends to a bilinear form $(\cdot \mid \cdot): \hat{\mathfrak{g}} \times \hat{\mathfrak{g}} \rightarrow \mathbb{C}$ which is given by the equations

$$
\begin{aligned}
\left(x \otimes t^{n} \mid y \otimes t^{m}\right) & =\delta_{n,-m} \kappa(x, y), \\
\left(c \mid \mathfrak{g} \otimes_{\mathbb{C}} \mathbb{C}\left[t, t^{-1}\right] \oplus \mathbb{C} c\right) & =\{0\}, \\
\left(d \mid \mathfrak{g} \otimes_{\mathbb{C}} \mathbb{C}\left[t, t^{-1}\right] \oplus \mathbb{C} d\right) & =\{0\}, \\
(c \mid d) & =1,
\end{aligned}
$$

for $x, y \in \mathfrak{g}$ and $m, n \in \mathbb{Z}$. It is nondegenerate, symmetric and invariant, i.e., $([x, y] \mid z)=(x \mid[y, z])$ for all $x, y, z \in \hat{\mathfrak{g}}$. Furthermore, it induces a nondegenerate bilinear form on the affine Cartan subalgebra and thus an isomorphism $v: \widehat{\mathfrak{h}} \sim \widehat{\mathfrak{h}}^{*}$ which coincides with the isomorphism $\mathfrak{h} \stackrel{\sim}{\longrightarrow} \mathfrak{h}^{*}$ induced by the Killing form, when restricted to the finite Cartan subalgebra, and which sends $c$ to $\delta$ and $d$ to $\Lambda_{\circ}$. So the induced form on $\widehat{\mathfrak{h}}^{*}$ is given by

$$
\begin{aligned}
(\alpha \mid \beta) & =\kappa(\alpha, \beta), \\
\left(\Lambda_{\circ} \mid \mathfrak{h}^{*} \oplus \mathbb{C} \Lambda_{\circ}\right) & =\{0\}, \\
\left(\delta \mid \mathfrak{h}^{*} \oplus \mathbb{C} \delta\right) & =\{0\}, \\
\left(\Lambda_{\circ} \mid \delta\right) & =1,
\end{aligned}
$$


for $\alpha, \beta \in \mathfrak{h}^{*}$ and $\kappa$ the induced Killing form on $\mathfrak{h}^{*}$. The induced form is invariant under the linear action of the affine Weyl group, i.e.,

$$
(w(\lambda) \mid w(\mu))=(\lambda \mid \mu)
$$

for $\lambda, \mu \in \widehat{\mathfrak{h}}^{*}, w \in \widehat{\mathscr{W}}$.

\section{Verma modules}

For a Lie algebra $\mathfrak{a}$ we denote by $U(\mathfrak{a})$ its universal enveloping algebra. For $\lambda \in \widehat{\mathfrak{h}}^{*}$ let $\mathbb{C}_{\lambda}$ be the one-dimensional representation of $U(\widehat{\mathfrak{b}})$ on which $\widehat{\mathfrak{h}}$ acts by the character $\lambda$ and $[\widehat{\mathfrak{b}}, \hat{\mathfrak{b}}]$ by zero. Then the Verma module with highest weight $\lambda$ is defined by

$$
\Delta(\lambda):=U(\hat{\mathfrak{g}}) \otimes_{U(\widehat{\mathfrak{b}})} \mathbb{C}_{\lambda} .
$$

It has a unique simple quotient, which we denote by $L(\lambda)$, and both modules are highest weight modules with highest weight $\lambda$.

Deformed Verma modules. Denote by $\widehat{S}:=S(\widehat{\mathfrak{h}})=U(\widehat{\mathfrak{h}})$ the symmetric algebra over the vector space $\hat{\mathfrak{h}}$ and by $S=S(\mathfrak{h})$ the symmetric algebra over the vector space $\mathfrak{h}$. Then the projection $\widehat{\mathfrak{h}} \rightarrow \mathfrak{h}$ induces a homomorphism $\widehat{S} \rightarrow S$ and equips $S$ with an $\widehat{S}$-algebra structure. We call a commutative, unital, noetherian $\widehat{S}$-algebra with structure morphism $\tau: \widehat{S} \rightarrow A$ a deformation algebra.

For a Lie algebra $\mathfrak{a}$ we set $\mathfrak{a}_{A}:=\mathfrak{a} \otimes_{\mathbb{C}} A$. Then we can identify $\left(\widehat{\mathfrak{h}}_{A}\right)^{*}=$ $\operatorname{Hom}_{A}\left(\widehat{\mathfrak{h}}_{A}, A\right)$ with $\widehat{\mathfrak{h}}^{*} \otimes_{\mathbb{C}} A$ and any weight $\lambda \in \widehat{\mathfrak{h}}^{*}$ induces a weight $\lambda \otimes 1 \in \widehat{\mathfrak{h}}_{A}^{*}$, which we simply denote by $\lambda$ again. In this way, the composition $\widehat{\mathfrak{h}} \hookrightarrow \widehat{S} \stackrel{\tau}{\rightarrow} A$ induces the canonical weight $\tau \in \widehat{\mathfrak{h}}_{A}^{*}$.

For $\lambda \in \widehat{\mathfrak{h}}^{*}$ let $A_{\lambda}$ be the $\widehat{\mathfrak{b}}_{A}$-module which is $A$ as an $A$-module and on which $\widehat{\mathfrak{h}}$ acts via the character $\lambda+\tau$ and $[\hat{\mathfrak{b}}, \hat{\mathfrak{b}}]$ by zero. We then define the deformed Verma module with highest weight $\lambda$ by

$$
\Delta_{A}(\lambda+\tau):=U\left(\hat{\mathfrak{g}}_{A}\right) \otimes_{U\left(\widehat{\mathfrak{b}}_{A}\right)} A_{\lambda} .
$$

For an $\widehat{\mathfrak{h}}_{A}$-module $M$ and $\lambda \in \widehat{\mathfrak{h}}^{*}$ we define the deformed weight space of $\lambda$ by

$$
M_{\lambda}:=\left\{m \in M \mid H m=(\lambda+\tau)(H) m \text { for all } H \in \widehat{\mathfrak{h}}_{A}\right\} .
$$

Then the deformed Verma module $\Delta_{A}(\lambda+\tau)$ decomposes as $\widehat{\mathfrak{h}}_{A}$-module into the direct sum of its weight spaces $\Delta_{A}(\lambda+\tau)_{\mu}$ with $\mu \in \widehat{\mathfrak{h}}^{*}$, such that $\Delta_{A}(\lambda+\tau)_{\mu} \neq 0$ implies $\mu \leq \lambda$.

If $A \rightarrow A^{\prime}$ is a homomorphism of deformation algebras with structure maps $\tau: \widehat{S} \rightarrow A$ and $\tau^{\prime}: \widehat{S} \rightarrow A \rightarrow A^{\prime}$, then

$$
\Delta_{A}(\lambda+\tau) \otimes_{A} A^{\prime} \cong \Delta_{A^{\prime}}\left(\lambda+\tau^{\prime}\right) .
$$


Note that for $\tau: \widehat{S} \rightarrow \mathbb{C}$, the surjection on the quotient $\mathbb{C} \cong \widehat{S} / \widehat{\mathfrak{h}} \widehat{S}$, we have $\Delta_{\mathbb{C}}(\lambda+\tau) \cong \Delta(\lambda)$.

Characters. Let $\mathbb{Z}\left[\widehat{\mathfrak{h}}^{*}\right]=\bigoplus \mathbb{Z} e^{\lambda}$ be the group algebra of $\widehat{\mathfrak{h}}^{*}$. We define a certain completion by $\lambda \in \widehat{\mathfrak{h}}^{*}$

$$
\widehat{\mathbb{Z}\left[\hat{\mathfrak{h}}^{*}\right]} \subset \prod_{\lambda \in \widehat{\mathfrak{h}}^{*}} \mathbb{Z} e^{\lambda}
$$

to be the subgroup of elements $\left(c_{\lambda}\right)$ with the property that there exists a finite subset $\left\{\mu_{1}, \ldots, \mu_{n}\right\} \subset \widehat{\mathfrak{h}}^{*}$ such that $c_{\lambda} \neq 0$ implies $\lambda \leq \mu_{i}$ for at least one $i$. Let $M \in \hat{\mathfrak{g}}$-mod be semisimple over $\widehat{\mathfrak{h}}$ with the properties that each weight space $M_{\lambda}$ is finite-dimensional and that there exists $\mu_{1}, \ldots, \mu_{n} \in \widehat{\mathfrak{h}}^{*}$ such that $M_{\lambda} \neq 0$ implies $\lambda \leq \mu_{i}$ for at least one $i$. We define the character of $M$ as element in $\widehat{\mathbb{Z}\left[\hat{\mathfrak{h}}^{*}\right]}$ given by the formal sum

$$
\operatorname{ch} M:=\sum_{\lambda \in \widehat{\mathfrak{h}}^{*}}\left(\operatorname{dim}_{\mathbb{C}} M_{\lambda}\right) e^{\lambda}
$$

We define the generalized Kostant partition function $\mathscr{P}: \mathbb{Z} \widehat{R}^{+} \rightarrow \mathbb{N}$ by

$$
\mathscr{P}(v):= \begin{cases}\operatorname{dim}_{\mathbb{C}} \Delta(0)_{v} & \text { if } v \in \mathbb{N} \widehat{R}^{+}, \\ 0 & \text { otherwise. }\end{cases}
$$

The name partition function comes from the combinatorial description of the formula

$$
\operatorname{ch} \Delta(\lambda)=\prod_{\alpha \in \hat{R}^{+}}\left(1+e^{-\alpha}+e^{-2 \alpha}+\cdots\right)^{\operatorname{dim} \hat{\mathfrak{g}}_{\alpha}}
$$

(compare [Kac 1990, Section 9.7]).

\section{Restricted Verma modules}

An equivalence relation. For a deformation algebra $A$ with canonical weight $\tau$ : $\widehat{\mathfrak{h}}_{A} \rightarrow A$, we extend the bilinear form $(\cdot \mid \cdot): \widehat{\mathfrak{h}}^{*} \times \widehat{\mathfrak{h}}^{*} \rightarrow \mathbb{C}$ to an $A$-linear continuation $(\cdot \mid \cdot)_{A}: \hat{\mathfrak{h}}_{A}^{*} \times \widehat{\mathfrak{h}}_{A}^{*} \rightarrow A$.

Let $A=\mathbb{K}$ be a field. For $v, \lambda \in \widehat{\mathfrak{h}}^{*}$ we write $v \preceq_{\mathbb{K}} \lambda$ if there exists $n \in \mathbb{N}$ and $\alpha \in \widehat{R}^{+}$such that $2(\lambda+\rho+\tau \mid \alpha)_{\mathbb{K}}=n(\alpha \mid \alpha)_{\mathbb{K}}$ and $v=\lambda-n \alpha$. We now denote by $\preceq_{\mathbb{K}}$ the partial ordering on $\widehat{\mathfrak{h}}^{*}$ which is generated by such tuples $v \preceq_{\mathbb{K}} \lambda$. Then $\preceq_{\mathbb{K}}$ is a refinement of the usual ordering $\leq$ on $\widehat{\mathfrak{h}}^{*}$. We denote by $\sim_{\mathbb{K}}$ the equivalence relation on $\widehat{\mathfrak{h}}^{*}$ which is generated by $\preceq_{\mathbb{K}}$.

Let $L_{\mathbb{K}}(\lambda+\tau)$ be the unique simple quotient of $\Delta_{\mathbb{K}}(\lambda+\tau)$ and denote by $\left[\Delta_{\mathbb{K}}(\lambda+\tau): L_{\mathbb{K}}(\mu+\tau)\right]$ the number of subquotients of a composition series of $\Delta_{\mathbb{K}}(\lambda+\tau)$ which are isomorphic to $L_{\mathbb{K}}(\mu+\tau)$. 
Theorem 4.1 [Kac and Kazhdan 1979, Theorem 2]. We have

$$
\left[\Delta_{\mathbb{K}}(\lambda+\tau): L_{\mathbb{K}}(\mu+\tau)\right] \neq 0
$$

if and only if $\mu \preceq_{\mathbb{K}} \lambda$.

Remark 4.2. From [Rocha-Caridi and Wallach 1982, Theorem 15] we know that $\left[\Delta_{\mathbb{K}}(\lambda+\tau): L_{\mathbb{K}}(\mu+\tau)\right] \neq 0$ if and only if there exists an embedding $\Delta_{\mathbb{K}}(\mu+\tau) \hookrightarrow$ $\Delta_{\mathbb{K}}(\lambda+\tau)$. Thus Theorem 4.1 also contains information about embeddings of Verma modules.

For $\lambda \in \widehat{\mathfrak{h}}^{*}$ we define the integral roots (with respect to $\lambda$ and $A$ ) by

$$
\widehat{R}_{A}(\lambda):=\left\{\alpha \in \widehat{R} \mid 2(\lambda+\rho+\tau \mid \alpha)_{A} \in \mathbb{Z}(\alpha \mid \alpha)_{A}\right\},
$$

and the corresponding integral Weyl group by

$$
\widehat{\mathscr{W}}_{A}(\lambda):=\left\langle s_{\alpha} \mid \alpha \in \widehat{R}_{A}(\lambda) \cap \widehat{R}^{\mathrm{re}}\right\rangle \subset \widehat{\mathscr{W}} .
$$

We write $\widehat{R}_{A}(\lambda)^{+}=\widehat{R}^{+} \cap \widehat{R}_{A}(\lambda)$ and $\hat{R}(\lambda)=\widehat{R}_{\mathbb{C}}(\lambda)$ in case $\tau: \hat{S} \rightarrow \widehat{S} / \widehat{\mathfrak{h}} \widehat{S} \cong \mathbb{C}$ is the quotient map and similarly $\widehat{\mathscr{W}}(\lambda)=\widehat{\mathscr{W}}_{\mathbb{C}}(\lambda)$.

The critical level. For $\lambda \in \widehat{\mathfrak{h}}^{*}$ we define the level of $\lambda$ to be the complex number $\lambda(c) \in \mathbb{C}$. If $\lambda \sim \mathbb{R} \mu$, we have $\lambda(c)=\mu(c)$. Therefore, the equivalence class $\Lambda$ of $\lambda$ has a well-defined level, and we have $v(c)=(v \mid \delta)$ for all $v \in \widehat{\mathfrak{h}}^{*}$.

Lemma 4.3 [Arakawa and Fiebig 2012b, Lemma 4.2]. For $\Lambda \in \widehat{\mathfrak{h}}^{*} / \sim_{\mathbb{K}}$ the following are equivalent.

(1) We have $\lambda(c)=-\rho(c)$ for some $\lambda \in \Lambda$.

(2) We have $\lambda(c)=-\rho(c)$ for all $\lambda \in \Lambda$.

(3) We have $\lambda+\delta \in \Lambda$ for all $\lambda \in \Lambda$.

(4) We have $n \delta \in \widehat{R}_{\mathbb{K}}(\lambda)$ for all $n \neq 0$ and $\lambda \in \Lambda$.

We call crit $:=-\rho(c)$ the critical level.

Denote by $\hat{\mathfrak{h}}_{\text {crit }}^{*}$ the hyperplane which consists of all $\lambda \in \widehat{\mathfrak{h}}^{*}$ with $\lambda(c)=$ crit. Then for each $\lambda \in \widehat{\mathfrak{h}}_{\text {crit }}^{*}$ we have $(\lambda+\rho \mid \delta)=0$.

Restricted Verma modules. Let $\lambda \in \widehat{\mathfrak{h}}^{*}$ and $\tau: \widehat{S} \rightarrow A$ be a deformation algebra which is an integral domain. Denote by $Q(A)$ its field of fractions and assume that both structure maps factor through the restriction map $\widehat{S} \rightarrow S$. This implies that $\tau(c)=\tau(d)=0$. We define $\Delta_{A}^{-}(\lambda+\tau)$ to be the submodule of $\Delta_{A}(\lambda+\tau)$ which is generated by the images of all homomorphisms $\Delta_{A}(\lambda-n \delta+\tau) \rightarrow \Delta_{A}(\lambda+\tau)$ for $n \in \mathbb{N}_{>0}$. Since $\tau(c)=\tau(d)=0$, we have $(\tau \mid \delta)=0$, and by Theorem 4.1 and its remark there is an injective map $\Delta_{Q(A)}(\lambda-n \delta+\tau) \hookrightarrow \Delta_{Q(A)}(\lambda+\tau)$ for every $n>0$ and $\lambda$ critical. But by our assumption on $A$, this also induces an injective 
map $\Delta_{A}(\lambda-n \delta+\tau) \hookrightarrow \Delta_{A}(\lambda+\tau)$. If $\lambda$ is noncritical, we get $\Delta_{A}^{-}(\lambda+\tau)=\{0\}$. We now define the restricted Verma module as the quotient

$$
\bar{\Delta}_{A}(\lambda+\tau)=\Delta_{A}(\lambda+\tau) / \Delta_{A}^{-}(\lambda+\tau) .
$$

As in the nonrestricted case, we omit the subscript of the restricted Verma modules if the deformation algebra is $\mathbb{C} \cong S / S \mathfrak{h}$. For example, we write $\bar{\Delta}(\lambda)$ instead of $\bar{\Delta}_{\mathbb{C}}(\lambda)$.

Remark 4.4. There is an alternative definition of restricted Verma modules. Denote by $V^{\text {crit }}(\mathfrak{g})$ the universal affine vertex algebra at the critical level and denote by $\mathfrak{z}$ its center. Then each smooth $[\hat{\mathfrak{g}}, \hat{\mathfrak{g}}]$-module $M$ carries the structure of a graded $\mathfrak{z}$-module. By a theorem of Feigin and Frenkel [1992], $\mathfrak{z}$ yields an action on $M$ by the graded polynomial ring generated by infinitely many homogeneous elements

$$
\mathscr{L}=\mathbb{C}\left[p_{s}^{(i)} \mid i=1, \ldots, l, s \in \mathbb{Z}\right]=\bigoplus_{n \in \mathbb{Z}} \mathscr{L}_{n} .
$$

Now a theorem of Frenkel and Gaitsgory [2006] shows that for any critical weight $\lambda \in \widehat{\mathfrak{h}}^{*}$ and $n<0$ there is a surjective map $\mathscr{L}_{n} \rightarrow \operatorname{Hom}_{\mathfrak{g}}(\Delta(\lambda+n \delta), \Delta(\lambda))$. Thus, the restricted Verma module $\bar{\Delta}(\lambda)$ coincides with the quotient

$$
\Delta(\lambda)^{\mathrm{res}}:=\Delta(\lambda) / \sum_{n<0} \mathscr{E}_{n} \Delta(\lambda) .
$$

However, we will not use this alternative description of restricted Verma modules in the rest of this paper.

Let $:: \widehat{\mathfrak{h}}^{*} \rightarrow \mathfrak{h}^{*}$ be the map induced by $\mathfrak{h} \hookrightarrow \widehat{\mathfrak{h}}$. For any subset $\Lambda \subset \widehat{\mathfrak{h}}^{*}$ we denote by $\bar{\Lambda} \subset \mathfrak{h}^{*}$ its image under .

Definition 4.5. Let $\Lambda \in \widehat{\mathfrak{h}}_{\text {crit }}^{*} / \sim_{\mathbb{K}}$ be a critical equivalence class. We call $\Lambda$

(1) generic if $\bar{\Lambda} \subset \mathfrak{h}^{*}$ contains exactly one element;

(2) subgeneric if $\bar{\Lambda} \subset \mathfrak{h}^{*}$ contains exactly two elements.

We call any weight contained in a generic (subgeneric, resp.) equivalence class a generic (subgeneric, resp.) weight. If $\Lambda$ is subgeneric, there is a weight $\bar{\lambda} \in \mathfrak{h}^{*}$ and a finite root $\alpha \in R$ such that $\bar{\Lambda}=\left\{\bar{\lambda}, s_{\alpha} \cdot \bar{\lambda}\right\}$.

Let $\lambda \in \widehat{\mathfrak{h}}_{\text {crit }}^{*}$ be a critical weight. Similarly to the integral roots of $\lambda$ we now define the finite integral root system (with respect to $\lambda$ and the deformation algebra $A$ ) by

$$
R_{A}(\lambda):=\widehat{R}_{A}(\lambda) \cap R=\left\{\alpha \in R \mid 2(\lambda+\rho+\tau \mid \alpha)_{A} \in \mathbb{Z}(\alpha \mid \alpha)_{A}\right\},
$$

and the finite integral Weyl group by

$$
\mathscr{W}_{A}(\lambda)=\widehat{\mathscr{W}}_{A}(\lambda) \cap \mathcal{W} .
$$


Again we write $R_{A}(\lambda)^{+}=R^{+} \cap R_{A}(\lambda)$ and $R(\lambda)=R_{\mathbb{C}}(\lambda)$ if the deformation algebra is $\mathbb{C}$. For $\lambda \in \widehat{\mathfrak{h}}_{\text {crit }}^{*}$ and $\alpha \in R_{A}(\lambda)$, such that $s_{\alpha} \cdot \lambda \neq \lambda$, we have either $s_{\alpha} \cdot \lambda<\lambda$ or $s_{-\alpha+\delta} \cdot \lambda<\lambda$. We define $\alpha \downarrow \lambda$ to be the element in the set $\left\{s_{\alpha} \cdot \lambda, s_{-\alpha+\delta} \cdot \lambda\right\}$ which is smaller than $\lambda$. Furthermore, we define inductively $\alpha \downarrow^{n} \lambda:=\alpha \downarrow\left(\alpha \downarrow^{n-1} \lambda\right)$. In case $s_{\alpha} \cdot \lambda=\lambda$ we have $\alpha \downarrow \lambda=\lambda$.

Now [2012b, Corollary 4.10] gives in our set up the following theorem:

Theorem 4.6. Let $\lambda \in \widehat{\mathfrak{h}}_{\text {crit }}^{*}$ and $\tau: \widehat{S} \rightarrow \mathbb{K}$ be a deformation algebra with $\mathbb{K}$ being a field. Assume that the structure map $\tau$ factors through $S$.

(1) If $\lambda$ is generic, $\bar{\Delta}_{\mathbb{K}}(\lambda+\tau)$ is simple.

(2) If $\lambda$ is subgeneric with $R_{\mathbb{K}}(\lambda)=\{ \pm \alpha\}$, we have a short exact sequence

$$
L_{\mathbb{K}}(\alpha \downarrow \lambda) \hookrightarrow \bar{\Delta}_{\mathbb{K}}(\lambda) \rightarrow L_{\mathbb{K}}(\lambda) .
$$

Note that the term subgeneric implies $\lambda \neq \alpha \downarrow \lambda$.

\section{The restricted Jantzen sum formula}

Andersen et al. [1994, Chapter 6] established a Jantzen sum formula for baby Verma modules. It relates the sum of the characters of the Jantzen filtration to an alternating sum of characters of baby Verma modules with smaller highest weights. We deduce a similar formula for the restricted Verma modules at the critical level. Theorem 5.1. Let $\lambda \in \widehat{\mathfrak{h}}_{\text {crit }}^{*}$. There is a filtration

$$
\bar{\Delta}(\lambda)=\bar{\Delta}(\lambda)^{0} \supset \bar{\Delta}(\lambda)^{1} \supset \bar{\Delta}(\lambda)^{2} \supset \cdots
$$

with these properties:

(1) $\bar{\Delta}(\lambda)^{1}$ is the maximal submodule of $\bar{\Delta}(\lambda)$.

(2) $\sum_{i>0} \operatorname{ch} \bar{\Delta}(\lambda)^{i}=\sum_{\alpha \in R(\lambda)^{+}}\left(\sum_{i>0}\left(\operatorname{ch} \bar{\Delta}\left(\alpha \downarrow^{2 i-1} \lambda\right)-\operatorname{ch} \bar{\Delta}\left(\alpha \downarrow^{2 i} \lambda\right)\right)\right)$.

Note that the sum is taken over all finite, positive, integral roots $\alpha \in R(\lambda)^{+}$.

The Shapovalov determinant. Let $\sigma: \hat{\mathfrak{g}} \rightarrow \hat{\mathfrak{g}}$ be a Chevalley-involution and define $\widehat{\mathfrak{n}}_{+}:=\bigoplus_{\alpha>0} \hat{\mathfrak{g}}_{\alpha}$ and $\widehat{\mathfrak{n}}_{-}:=\bigoplus_{\alpha<0} \hat{\mathfrak{g}}_{\alpha}$, where $\widehat{\mathfrak{g}}_{\alpha}$ is the root space of $\hat{\mathfrak{g}}$ corresponding to the $\operatorname{root} \alpha \in \hat{R}$. There is a decomposition $U(\widehat{\mathfrak{g}})=U(\widehat{\mathfrak{h}}) \oplus\left(\widehat{\mathfrak{n}}_{-} U(\widehat{\mathfrak{g}})+U(\widehat{\mathfrak{g}}) \widehat{\mathfrak{n}}_{+}\right)$, and we denote by $\beta: U(\widehat{\mathfrak{g}}) \rightarrow S(\widehat{\mathfrak{h}})$ the projection to the first summand of this decomposition.

The Shapovalov form is now defined as the bilinear pairing $F: U(\widehat{\mathfrak{g}}) \times U(\widehat{\mathfrak{g}}) \rightarrow S(\widehat{\mathfrak{h}})$ with $F(x, y)=\beta(\sigma(x) y)$. It is symmetric and contravariant, i.e., for $u, x, y \in U(\hat{\mathfrak{g}})$ we have $F(\sigma(u) x, y)=F(x, u y)$. For $\eta \in \mathbb{N} \widehat{R}^{+}$we denote by $F_{\eta}$ the restriction of $F$ to the weight space $U\left(\widehat{\mathfrak{n}}_{-}\right)_{-\eta}$. Recall the isomorphism $v: \widehat{\mathfrak{h}} \sim \widehat{\mathfrak{h}}^{*}$ induced by the bilinear form $(\cdot \mid \cdot)$ on $\widehat{\mathfrak{h}}$ and define $h_{\alpha}:=v^{-1}(\alpha)$ for any root $\alpha \in \widehat{R}$. 
Theorem 5.2 [Kac and Kazhdan 1979, Theorem 1]. The determinant of

$$
F_{\eta}: U\left(\widehat{\mathfrak{n}}_{-}\right)_{-\eta} \times U\left(\widehat{\mathfrak{n}}_{-}\right)_{-\eta} \rightarrow S(\widehat{\mathfrak{h}})
$$

is, up to multiplication with a nonzero complex number, given by the formula

$$
\operatorname{det} F_{\eta}=\prod_{\alpha \in \hat{R}^{+}} \prod_{n=1}^{\infty}\left(h_{\alpha}+\rho\left(h_{\alpha}\right)-n \frac{(\alpha \mid \alpha)}{2}\right)^{\operatorname{mult}(\alpha) \cdot \mathscr{P}(\eta-n \alpha)},
$$

where $\mathscr{P}$ is Kostant's partition function and $\operatorname{mult}(\alpha):=\operatorname{dim}_{\mathbb{C}}\left(\widehat{\mathfrak{g}}_{\alpha}\right)$.

We equip the polynomial ring $\mathbb{C}[t]$ in one variable with two different structures of a deformation algebra. The first one is given by the map $\tau_{1}: \hat{S} \rightarrow \mathbb{C}[t]$, where $\tau_{1}$ is induced by the inclusion of the line $\mathbb{C} \rho \subset \widehat{\mathfrak{h}}^{*}$. The second $\widehat{S}$-module structure $\tau_{2}: \hat{S} \rightarrow \mathbb{C}[t]$ is given by the inclusion $\mathbb{C} \bar{\rho} \subset \widehat{\mathfrak{h}}^{*}$. Recall that we consider elements of $\mathfrak{h}^{*}$ as elements of $\widehat{\mathfrak{h}}^{*}$ by the embedding from above. Furthermore, $\bar{\rho} \in \mathfrak{h}^{*}$ implies that $\tau_{2}$ factors through the restriction map $\widehat{S} \rightarrow S$. For a more intuitive notation, we follow [Jantzen 1979] and define $\Delta_{\mathbb{C}[t]}(\lambda+t \rho):=\Delta_{\mathbb{C}[t]}\left(\lambda+\tau_{1}\right)$ and $\Delta_{\mathbb{C}[t]}(\lambda+t \bar{\rho}):=\Delta_{\mathbb{C}[t]}\left(\lambda+\tau_{2}\right)$.

Note that for $\lambda \in \widehat{\mathfrak{h}}_{\text {crit }}^{*}$ critical, and since $\tau_{2}(c)=\tau_{2}(d)=t \bar{\rho}(c)=0$, we can construct the restricted Verma module $\bar{\Delta}_{\mathbb{C}[t]}(\lambda+t \bar{\rho})$. Let $\mathbb{C}(t)$ be the quotient field of $\mathbb{C}[t]$.

Lemma 5.3. Let $\lambda \in \widehat{\mathfrak{h}}_{\text {crit }}^{*}$ Then $\bar{\Delta}_{\mathbb{C}(t)}(\lambda+t \bar{\rho})=\bar{\Delta}_{\mathbb{C}[t]}(\lambda+t \bar{\rho}) \otimes_{\mathbb{C}[t]} \mathbb{C}(t)$ is simple.

Proof. If we prove that $R_{\mathbb{C}(t)}(\lambda)=\varnothing$, the lemma follows from Theorem 4.6. But since $(\bar{\rho} \mid \alpha) \neq 0$ for all $\alpha \in R^{+}$, we get $2(\lambda+\rho+t \bar{\rho} \mid \alpha)_{\mathbb{C}(t)} \notin \mathbb{Z}(\alpha \mid \alpha)_{\mathbb{C}(t)} \subset \mathbb{C}$ for all $\alpha \in R^{+}$.

The Shapovalov form induces symmetric, contravariant bilinear forms on $\Delta_{\widehat{S}}\left(\lambda+\epsilon^{\prime}\right)$ and $\bar{\Delta}_{S}(\lambda+\epsilon)$, where $\epsilon^{\prime} \in \widehat{\mathfrak{h}}_{\widehat{S}}^{*}$ denote the canonical weight induced by $\widehat{\mathfrak{h}} \hookrightarrow \widehat{S}$ and $\epsilon \in \widehat{\mathfrak{h}}_{S}^{*}$ denotes its composition with $\widehat{S} \rightarrow S$. Moreover, it induces contravariant forms on all Verma modules $\Delta_{\mathbb{C}[t]}(\lambda+t \rho), \bar{\Delta}_{\mathbb{C}[t]}(\lambda+t \bar{\rho})$, $\Delta_{\mathbb{C}[t]}(\lambda+t \bar{\rho}), \Delta(\lambda)$ and $\bar{\Delta}(\lambda)$, which we have to deal with in the rest of this paper. The contravariance of the forms implies for $\Delta(\lambda)$ and $\bar{\Delta}(\lambda)$ that the radicals of the forms coincide with the maximal submodules of $\Delta(\lambda)$ and $\bar{\Delta}(\lambda)$.

Restricted Jantzen filtration. Let $(\cdot, \cdot)$ be the contravariant form on $\bar{\Delta}_{\mathbb{C}[t]}(\lambda+t \bar{\rho})$ induced by the Shapovalov form. We first define a filtration on $\bar{\Delta}_{\mathbb{C}[t]}(\lambda+t \bar{\rho})$ by

$$
\bar{\Delta}_{\mathbb{C}[t]}(\lambda+t \bar{\rho})^{i}:=\left\{m \in \bar{\Delta}_{\mathbb{C}[t]}(\lambda+t \bar{\rho}) \mid\left(m, \bar{\Delta}_{\mathbb{C}[t]}(\lambda+t \bar{\rho})\right) \subset t^{i} \mathbb{C}[t]\right\} .
$$

The Jantzen filtration on $\bar{\Delta}(\lambda)$ is then defined by

$$
\bar{\Delta}(\lambda)^{i}:=\operatorname{im}\left(\bar{\Delta}_{\mathbb{C}[t]}(\lambda+t \bar{\rho})^{i} \hookrightarrow \bar{\Delta}_{\mathbb{C}[t]}(\lambda+t \bar{\rho}) \rightarrow \bar{\Delta}(\lambda)\right),
$$


where the second map is the specialization $t \mapsto 0$. In the same way we get the Jantzen filtration on $\Delta(\lambda)$ as it is defined in [Kac and Kazhdan 1979] using the deformed Verma module $\Delta_{\mathbb{C}[t]}(\lambda+t \rho)$.

Notation 5.4. Let $\mu \leq \lambda$. We denote the determinants of the contravariant bilinear forms on the $\mu$-weight spaces $\Delta_{\mathbb{C}[t]}(\lambda+t \bar{\rho})_{\mu}, \Delta_{\mathbb{C}[t]}(\lambda+t \rho)_{\mu}$ and $\bar{\Delta}_{\mathbb{C}[t]}(\lambda+t \bar{\rho})_{\mu}$ by $D_{\lambda+t \bar{\rho}}(\mu+t \bar{\rho}), D_{\lambda+t \rho}(\mu+t \rho)$ and $\bar{D}_{\lambda+t \bar{\rho}}(\mu+t \bar{\rho})$.

For a polynomial $P \in \mathbb{C}[t]$ denote by $\operatorname{ord}_{t}(P)$ the natural number $n \in \mathbb{N}$ with $t^{n} \mid P$ but $t^{n+1} \nmid P$.

Lemma 5.5. For the Jantzen filtrations of the $\mu$-weight spaces of the nonrestricted and restricted Verma modules we have the formulas

$$
\sum_{i>0} \operatorname{dim}_{\mathbb{C}} \Delta(\lambda)_{\mu}^{i}=\operatorname{ord}_{t}\left(D_{\lambda+t \rho}(\mu+t \rho)\right)
$$

and

$$
\sum_{i>0} \operatorname{dim}_{\mathbb{C}} \bar{\Delta}(\lambda)_{\mu}^{i}=\operatorname{ord}_{t}\left(\bar{D}_{\lambda+t \bar{\rho}}(\mu+t \bar{\rho})\right) .
$$

Proof. By Lemma 5.3 the Shapovalov form of the restricted deformed Verma module $\bar{\Delta}_{\mathbb{C}[t]}(\lambda)$ is nondegenerate. Thus we can apply [Jantzen 1979, Lemma 5.1] to get both formulas.

We first want to describe the Jantzen filtration of a restricted Verma module with a highest weight, which is critical and subgeneric.

Proposition 5.6 [Kübel 2014, Lemma 11]. Let $\lambda \in \widehat{\mathfrak{h}}_{\text {crit }}^{*}$ be subgeneric; that is, $R(\lambda)=\{ \pm \alpha\}$ for a finite positive root $\alpha \in R^{+}$and $\alpha \downarrow \lambda \neq \lambda$. Then the Jantzen filtration of $\bar{\Delta}(\lambda)$ is

$$
\bar{\Delta}(\lambda) \supset L(\alpha \downarrow \lambda) \supset 0,
$$

and we have the alternating sum formula

$$
\sum_{i>0} \operatorname{ch} \bar{\Delta}(\lambda)^{i}=\operatorname{ch} L(\alpha \downarrow \lambda)=\operatorname{ch} \bar{\Delta}(\alpha \downarrow \lambda)-\operatorname{ch} \bar{\Delta}\left(\alpha \downarrow^{2} \lambda\right)+\operatorname{ch} \bar{\Delta}\left(\alpha \downarrow^{3} \lambda\right)-\cdots .
$$

Proof. The first part of the proposition is [Kübel 2014, Lemma 11]. The second part follows inductively from Theorem 4.6.

Recall the canonical weight $\epsilon: \widehat{\mathfrak{h}}_{S} \rightarrow S$ induced by $\widehat{\mathfrak{h}} \subset \widehat{S} \rightarrow S$, and for $\nu \geq 0$ denote by $\bar{D}_{\epsilon-\rho}(\epsilon-\rho-v)$ the determinant of the contravariant form on $\bar{\Delta}_{S}(\epsilon-\rho)_{-\rho-\nu}$. Let $\phi: S \rightarrow \mathbb{C}[t]$ be the algebra homomorphism given by

$$
\phi(H):=(\lambda+\rho)(H)+t \bar{\rho}(H)
$$


for all $H \in \mathfrak{h}$. If $p \in S$ is a prime element and $a \in S$, we denote by $\operatorname{ord}_{p}(a)$ the integer $n \in \mathbb{N}$ such that $p^{n} \mid a$ but $p^{n+1} \nmid a$. By [Jantzen 1979, Chapter 5.6], we get for $a \in S$

$$
\operatorname{ord}_{t}(\phi(a))=\sum_{p} \operatorname{ord}_{p}(a) \operatorname{ord}_{t}(\phi(p))
$$

where $p$ runs over all classes of associated prime elements of $S$. As in Lemma 5.3 we see that, for the quotient field $Q=Q(S)$ of $S$, the restricted Verma module $\bar{\Delta}_{Q}(\epsilon-\rho) \cong \bar{\Delta}_{S}(\epsilon-\rho) \otimes_{S} Q$ is simple. We conclude that $\bar{D}_{\epsilon-\rho}(\epsilon-\rho-v) \neq 0$ and also $\phi\left(\bar{D}_{\epsilon-\rho}(\epsilon-\rho-v)\right) \neq 0$ for all $v \in \mathbb{N} \widehat{R}^{+}$. Combining (5-1) with Lemma 5.5, we get

$$
\sum_{n>0} \operatorname{ch} \bar{\Delta}(\lambda)^{n}=e^{\lambda} \sum_{p} \operatorname{ord}_{t}(\phi(p)) \sum_{\nu \in \mathbb{N}^{+}} \operatorname{ord}_{p}\left(\bar{D}_{\epsilon-\rho}(\epsilon-\rho-v)\right) e^{-v} .
$$

We are now able to prove the general case. We follow [Jantzen 1979, Chapter 5.7]. Proof of Theorem 5.1. If $\lambda \in \widehat{\mathfrak{h}}_{\text {crit }}^{*}$ fulfills $\left\langle\lambda+\rho, \alpha^{\vee}\right\rangle \notin \mathbb{Z} \backslash\{0\}$ for any finite positive root $\alpha \in R^{+}$, then $\lambda$ is a generic weight and $\bar{\Delta}(\lambda)$ is simple, by Theorem 4.6. The evaluation of the polynomial $\bar{D}_{\epsilon-\rho}(\epsilon-\rho-v) \in S$ at $\lambda+\rho$ for $v \in \mathbb{N} \widehat{R}^{+}$can be viewed as the determinant of the contravariant form on the weight space $\bar{\Delta}(\lambda)_{\lambda-v}$ induced by the Shapovalov form. Since the weight spaces are orthogonal to each other according to the contravariant form, $\bar{D}_{\epsilon-\rho}(\epsilon-\rho-v)(\lambda+\rho)$ is unequal to zero for all $v \in \mathbb{N} \widehat{R}^{+}$. Otherwise we could construct a proper submodule of $\bar{\Delta}(\lambda)$, which would be a contradiction. $\bar{D}_{\epsilon-\rho}(\epsilon-\rho-\nu)$ decomposes into a product of linear factors, and it follows that all prime divisors of $\bar{D}_{\epsilon-\rho}(\epsilon-\rho-v)$ are of the form $\alpha^{\vee}-r$, where $\alpha \in R^{+}$and $r \in \mathbb{Z} \backslash\{0\}$.

For $\alpha \in R^{+}$and $r \in \mathbb{Z}$ we define $v_{\alpha, r} \in \mathbb{Z}\left[\hat{\mathfrak{h}}^{*}\right]$ by

$$
v_{\alpha, r}=\sum_{\eta \in \mathbb{N}^{+}} \operatorname{ord}_{\alpha^{\vee}-r}\left(\bar{D}_{\epsilon-\rho}(\epsilon-\rho-\eta)\right) e^{-\eta} .
$$

Because $\left\langle\bar{\rho}, \alpha^{\vee}\right\rangle \neq 0$ for any $\alpha \in R^{+}$, the restriction of $\alpha^{\vee}-r$ to the curve $(\lambda+\rho)+\mathbb{C} \bar{\rho} \subset \widehat{\mathfrak{h}}^{*}$ is unequal to zero, i.e., in formulas we have

$$
\phi\left(\alpha^{\vee}-r\right)=\left\langle\lambda+\rho, \alpha^{\vee}\right\rangle-r+t\left\langle\bar{\rho}, \alpha^{\vee}\right\rangle \neq 0 .
$$

If $r \neq\left\langle\lambda+\rho, \alpha^{\vee}\right\rangle$, then $\operatorname{ord}_{t}\left(\left\langle\lambda+\rho, \alpha^{\vee}\right\rangle-r+t\left\langle\bar{\rho}, \alpha^{\vee}\right\rangle\right)=0$. However, for $r=\left\langle\lambda+\rho, \alpha^{\vee}\right\rangle$ we have $\operatorname{ord}_{t}\left(\left\langle\lambda+\rho, \alpha^{\vee}\right\rangle-r+t\left\langle\bar{\rho}, \alpha^{\vee}\right\rangle\right)=1$. Now $\alpha^{\vee}-\left\langle\lambda+\rho, \alpha^{\vee}\right\rangle$ can only be a prime divisor of $\bar{D}_{\epsilon-\rho}(\epsilon-\rho-\eta)$ if $\alpha \in R(\lambda)^{+}$. Applying formula (5-2) we conclude that

$$
\sum_{i>0} \operatorname{ch} \bar{\Delta}(\lambda)^{i}=\sum_{\alpha \in R(\lambda)^{+}} v_{\alpha,\left\langle\lambda+\rho, \alpha^{\vee}\right\rangle} e^{\lambda} .
$$


Let $\alpha \in R(\lambda)^{+}$. Perturbing the weight $\lambda$ in the hyperplane that contains $\lambda$ and is parallel to the reflection hyperplane corresponding to $\alpha$, we find a weight $\mu \in \widehat{\mathfrak{h}}_{\text {crit }}^{*}$ such that $\left\langle\mu+\rho, \alpha^{\vee}\right\rangle=n=\left\langle\lambda+\rho, \alpha^{\vee}\right\rangle$ and $\left\langle\mu+\rho, \beta^{\vee}\right\rangle \notin \mathbb{Z}$ for all $\beta \in R(\lambda)^{+} \backslash\{\alpha\}$. Thus, we've found a subgeneric weight $\mu$ with $R(\mu)=\{ \pm \alpha\}$ and $v_{\alpha,\left\langle\mu+\rho, \alpha^{\vee}\right\rangle}=$ $v_{\alpha,\left\langle\lambda+\rho, \alpha^{\vee}\right\rangle}$. But by Proposition 5.6, the Jantzen filtration of $\bar{\Delta}(\mu)$ is given by

$$
\bar{\Delta}(\mu) \supset L(\alpha \downarrow \mu) \supset 0 .
$$

We conclude using (5-3):

$$
v_{\alpha, n} e^{\mu}=\operatorname{ch} L(\alpha \downarrow \mu)=\sum_{i>0}\left(\operatorname{ch} \bar{\Delta}\left(\alpha \downarrow^{2 i-1} \mu\right)-\operatorname{ch} \bar{\Delta}\left(\alpha \downarrow^{2 i} \mu\right)\right) .
$$

Now the choice of $\mu$ implies that $e^{\lambda-\mu} \operatorname{ch} \bar{\Delta}\left(\alpha \downarrow^{n} \mu\right)=\operatorname{ch} \bar{\Delta}\left(\alpha \downarrow^{n} \lambda\right)$. Thus, we conclude

$$
v_{\alpha, n} e^{\lambda}=\sum_{i>0}\left(\operatorname{ch} \bar{\Delta}\left(\alpha \downarrow^{2 i-1} \lambda\right)-\operatorname{ch} \bar{\Delta}\left(\alpha \downarrow^{2 i} \lambda\right)\right) .
$$

Since we can apply this to any root $\beta \in R(\lambda)^{+}$we can use (5-3) once more to get the formula in Theorem 5.1.

As a consequence of Theorem 5.1 we get the linkage principle for restricted Verma modules at the critical level in the same way as in [Andersen et al. 1994, Chapter 6] or [Kumar and Letzter 1997, Theorem 10.3]. The linkage principle was already proved in [Arakawa and Fiebig 2012a] introducing restricted projective objects in the restricted category 0 over the Lie algebra $\hat{\mathfrak{g}}$. Our proof, however, avoids the rather complicated construction of restricted projective objects.

Corollary 5.7 [Arakawa and Fiebig 2012a, Theorem 5.1]. Let $\lambda \in \widehat{\mathfrak{h}}_{\text {crit }}^{*}$ and $\mu \in \widehat{\mathfrak{h}}^{*}$. Then $[\bar{\Delta}(\lambda): L(\mu)] \neq 0$ implies $\mu \in \widehat{\mathscr{W}}(\lambda) \cdot \lambda$ and $\mu \leq \lambda$.

Proof. The statement is obvious for $\lambda=\mu$, and it is also clear that $[\bar{\Delta}(\lambda): L(\mu)] \neq 0$ implies $\mu \leq \lambda$. We use induction on $\lambda-\mu$ and assume $\mu<\lambda$. If $[\bar{\Delta}(\lambda): L(\mu)] \neq 0$, then also $\left[\bar{\Delta}(\lambda)^{1}: L(\mu)\right] \neq 0$ since $\bar{\Delta}(\lambda)^{1} \subset \bar{\Delta}(\lambda)$ is the maximal submodule. But then the restricted Jantzen sum formula implies that $L(\mu)$ has to appear as a subquotient in some $\bar{\Delta}\left(\alpha \downarrow^{n} \lambda\right)$, where $\alpha \in R(\lambda)^{+}$and $n>0$. Our induction assumption then implies $\mu \in \widehat{W}\left(\alpha \downarrow^{n} \lambda\right) \cdot\left(\alpha \downarrow^{n} \lambda\right)$, but the definition of $\alpha \downarrow \lambda$ implies $\widehat{\mathscr{W}}\left(\alpha \downarrow^{n} \lambda\right) \cdot\left(\alpha \downarrow^{n} \lambda\right)=\widehat{\mathscr{W}}(\lambda) \cdot(\lambda)$.

\section{Acknowledgements}

I would like to thank Peter Fiebig for drawing my attention to the calculation of the above Jantzen sum formula and I am very grateful for his patience in explaining me the underlying theory. I also want to thank the referee for his valuable suggestions and comments to improve this article. 


\section{References}

[Andersen et al. 1994] H. H. Andersen, J. C. Jantzen, and W. Soergel, Representations of quantum groups at a pth root of unity and of semisimple groups in characteristic $p$ : independence of $p$, Astérisque 220, Société Mathématique de France, Paris, 1994. MR 95j:20036 Zbl 0802.17009

[Arakawa and Fiebig 2012a] T. Arakawa and P. Fiebig, "The linkage principle for restricted critical level representations of affine Kac-Moody algebras", Compos. Math. 148:6 (2012), 1787-1810. MR 2999304 Zbl 06147343

[Arakawa and Fiebig 2012b] T. Arakawa and P. Fiebig, "On the restricted Verma modules at the critical level”, Trans. Amer. Math. Soc. 364:9 (2012), 4683-4712. MR 2922606 Zbl 06191426

[Feigin and Frenkel 1992] B. Feigin and E. Frenkel, "Affine Kac-Moody algebras at the critical level and Gelfand-Dikii algebras", pp. 197-215 in Infinite analysis, Part A (Kyoto, 1991), edited by A. Tsuchiya et al., Adv. Ser. Math. Phys. 16, World Scientific, River Edge, NJ, 1992. MR 93j:17049 Zbl 0925.17022

[Frenkel 2005] E. Frenkel, "Wakimoto modules, opers and the center at the critical level", Adv. Math. 195:2 (2005), 297-404. MR 2006d:17018 Zbl 1129.17014

[Frenkel and Gaitsgory 2006] E. Frenkel and D. Gaitsgory, "Local geometric Langlands correspondence and affine Kac-Moody algebras", pp. 69-260 in Algebraic geometry and number theory, edited by V. Ginzburg, Progr. Math. 253, Birkhäuser, Boston, MA, 2006. MR 2008e:17023 Zbl 1184.17011

[Jantzen 1979] J. C. Jantzen, Moduln mit einem höchsten Gewicht, Lecture Notes in Mathematics 750, Springer, Berlin, 1979. MR 81m:17011 Zbl 0426.17001

[Kac 1990] V. G. Kac, Infinite-dimensional Lie algebras, 3rd ed., Cambridge University Press, 1990. MR 92k:17038 Zbl 0716.17022

[Kac and Kazhdan 1979] V. G. Kac and D. A. Kazhdan, "Structure of representations with highest weight of infinite-dimensional Lie algebras”, Adv. in Math. 34:1 (1979), 97-108. MR 81d:17004 Zbl 0427.17011

[Kübel 2014] J. Kübel, "Centers for the restricted category 0 at the critical level over affine KacMoody algebras”, Math. Z. 276:3-4 (2014), 1133-1149. MR 3175174

[Kumar and Letzter 1997] S. Kumar and G. Letzter, "Shapovalov determinant for restricted and quantized restricted enveloping algebras", Pacific J. Math. 179:1 (1997), 123-161. MR 98g:17014 Zbl 0965.17006

[Rocha-Caridi and Wallach 1982] A. Rocha-Caridi and N. R. Wallach, "Projective modules over graded Lie algebras, I”, Math. Z. 180:2 (1982), 151-177. MR 83h:17018 Zbl 0467.17006

Received January 28, 2013. Revised July 15, 2013.

JOHANNES KÜBEL

DEPARTMENT OF MATHEMATICS

UNIVERSITY OF ERLANGEN

CAUERSTR. 11

91058 ERLANGEN

GERMANY

kuebel@mi.uni-erlangen.de 


\title{
PACIFIC JOURNAL OF MATHEMATICS
}

\author{
msp.org/pjm
}

Founded in 1951 by E. F. Beckenbach (1906-1982) and F. Wolf (1904-1989)

\section{EDITORS}

Don Blasius (Managing Editor)

Department of Mathematics

University of California

Los Angeles, CA 90095-1555

blasius@math.ucla.edu

\author{
Paul Balmer \\ Department of Mathematics \\ University of California \\ Los Angeles, CA 90095-1555 \\ balmer@math.ucla.edu \\ Robert Finn \\ Department of Mathematics \\ Stanford University \\ Stanford, CA 94305-2125 \\ finn@math.stanford.edu \\ Sorin Popa \\ Department of Mathematics \\ University of California \\ Los Angeles, CA 90095-1555 \\ popa@math.ucla.edu
}

\author{
Vyjayanthi Chari \\ Department of Mathematics \\ University of California \\ Riverside, CA 92521-0135 \\ chari@math.ucr.edu \\ Kefeng Liu \\ Department of Mathematics \\ University of California \\ Los Angeles, CA 90095-1555 \\ liu@math.ucla.edu \\ Jie Qing \\ Department of Mathematics \\ University of California \\ Santa Cruz, CA 95064 \\ qing@ cats.ucsc.edu
}

\section{PRODUCTION}

Silvio Levy, Scientific Editor, production@msp.org

\section{SUPPORTING INSTITUTIONS}

ACADEMIA SINICA, TAIPEI

CALIFORNIA INST. OF TECHNOLOGY

INST. DE MATEMÁTICA PURA E APLICADA

KEIO UNIVERSITY

MATH. SCIENCES RESEARCH INSTITUTE

NEW MEXICO STATE UNIV.

OREGON STATE UNIV.

\author{
STANFORD UNIVERSITY \\ UNIV. OF BRITISH COLUMBIA \\ UNIV. OF CALIFORNIA, BERKELEY \\ UNIV. OF CALIFORNIA, DAVIS \\ UNIV. OF CALIFORNIA, LOS ANGELES \\ UNIV. OF CALIFORNIA, RIVERSIDE \\ UNIV. OF CALIFORNIA, SAN DIEGO \\ UNIV. OF CALIF., SANTA BARBARA
}

\author{
Daryl Cooper \\ Department of Mathematics \\ University of California \\ Santa Barbara, CA 93106-3080 \\ cooper@math.ucsb.edu \\ Jiang-Hua Lu \\ Department of Mathematics \\ The University of Hong Kong \\ Pokfulam Rd., Hong Kong \\ jhlu@maths.hku.hk \\ Paul Yang \\ Department of Mathematics \\ Princeton University \\ Princeton NJ 08544-1000 \\ yang@math.princeton.edu
}

These supporting institutions contribute to the cost of publication of this Journal, but they are not owners or publishers and have no responsibility for its contents or policies.

See inside back cover or msp.org/pjm for submission instructions.

The subscription price for 2014 is US $\$ 410 /$ year for the electronic version, and \$535/year for print and electronic.

Subscriptions, requests for back issues and changes of subscribers address should be sent to Pacific Journal of Mathematics, P.O. Box 4163, Berkeley, CA 94704-0163, U.S.A. The Pacific Journal of Mathematics is indexed by Mathematical Reviews, Zentralblatt MATH, PASCAL CNRS Index, Referativnyi Zhurnal, Current Mathematical Publications and Web of Knowledge (Science Citation Index).

The Pacific Journal of Mathematics (ISSN 0030-8730) at the University of California, c/o Department of Mathematics, 798 Evans Hall \#3840, Berkeley, CA 94720-3840, is published twelve times a year. Periodical rate postage paid at Berkeley, CA 94704, and additional mailing offices. POSTMASTER: send address changes to Pacific Journal of Mathematics, P.O. Box 4163, Berkeley, CA 94704-0163.

PJM peer review and production are managed by EditFLOW ${ }^{\circledR}$ from Mathematical Sciences Publishers.

\section{PUBLISHED BY}

\section{mathematical sciences publishers \\ nonprofit scientific publishing}

http://msp.org/

(C) 2014 Mathematical Sciences Publishers 


\section{PACIFIC JOURNAL OF MATHEMATICS}

Volume $269 \quad$ No. $2 \quad$ June 2014

Totaro's question for simply connected groups of low rank

257

JODI BLACK and RAMAN PARIMALA

Uniform hyperbolicity of the curve graphs

269

BRIAN H. BOWDITCH

Constant Gaussian curvature surfaces in the 3-sphere via loop groups

281

DAVID BRANDER, JUN-ICHI INOGUCHI and SHIMPEI KOBAYASHI

On embeddings into compactly generated groups

305

PIERRE-EMmanuel CAPRACE and Yves CORNULIER

Variational representations for $N$-cyclically monotone vector fields

ALFRED GALICHON and NASSIF GHOUSSOUB

Restricted successive minima

341

MaRTIN HenK and CARSTEN THIEL

Radial solutions of non-Archimedean pseudodifferential equations

355

ANATOLY N. KochubeI

A Jantzen sum formula for restricted Verma modules over affine Kac-Moody

algebras at the critical level

JOHANNES KÜBEL

Notes on the extension of the mean curvature flow

YAN LENG, ENTAO ZHAO and HaORAN ZHAO

Hypersurfaces with prescribed angle function

Henrique F. DE Lima, ERAldo A. Lima JR. and Ulisses L. Parente

Existence of nonparametric solutions for a capillary problem in warped products

JoRGE H. LiRA and GABRIELA A. WANDERLEY

A counterexample to the simple loop conjecture for $\operatorname{PSL}(2, \mathbb{R})$

425

KATHRYN MANN

Twisted Alexander polynomials of 2-bridge knots for parabolic representations

TAKAYUKI MORIFUJI and ANH T. TRAN

Schwarzian differential equations associated to Shimura curves of genus zero

FANG-TING TU

Polynomial invariants of Weyl groups for Kac-Moody groups 